\title{
PERCEPTION OF CULTURED MEAT AMONG SRI LANKAN COMMUNITY
}

\author{
Chandimala U.R.*, Gunathilake D.M.C.C., Vidanapathirana N.P. and Rifnas L.M. \\ Institute for Agro-Technology and Rural Sciences, Weligatta-New Town, Hambantota, Sri Lanka \\ *Corresponding Author: rushanthi@uciars.cmb.ac.lk (iD https://orcid.org/0000-0002-0647-0615) \\ Received: 25.12.2021; Accepted:19.01.2022; Published: 31.01.2022
}

\begin{abstract}
Production of cultured meat involves synthesizing meat from animal tissue samples in a controlled environment using biotechnology. This study was directed to understand the perception of Sri Lankan consumer on cultured meat consumption. A structural survey was conducted in Sri Lanka using a probability sample of 14200 people from 11 districts and it was selected proportionately to the population in selected divisional secretariat divisions of these districts. The result of the survey revealed that $75 \%$ of the population was interested in accepting the cultured meat. The study showed that the meat consumers usually considered the health benefits mostly followed by nutritional properties, sensory properties especially the flavor and texture and meat price. Participants have understood the advantages of cultured meat in terms of animal welfare, environmental aspects, and food security of the country. Therefore, it can be concluded that there will be a substantial market for cultured meat, when it is introduced to the market. Educating people about intrinsic properties of cultured meat will also be important. In addition, the regression analysis showed that the acceptance level of cultured meat increased with the education level of consumers.
\end{abstract}

\section{Keywords: Animal welfare, Cultured meat, Food security, Lab-grown meat}

\section{INTRODUCTION}

Meat which is a major source of proteins has seized a dominant position in Western food culture (Holmand and Mohl, 2000) since long ago. Globally, the demand for meat is increasing and even in vegetarian cultures, some dishes are made imitating the properties of meat considering the dominant nature of meat in cookery. But the plantbased meat substitutes known as protein analogues haven't still succeeded in replacing meat in human diet (Holmand and Mohl, 2000; Stephans et al., 2018). According to Rorheim et al., (2016), more than 60 billion animals are nurtured industrially in each year with the purpose of meat production. The global demand for meat is predicted to rise by $73 \%$ by year 2050 even though the meat industry is the globe's main cause of human pandemic diseases (Rorheim et al., 2016).

In Sri Lanka, even though the meat industry is having the influence of religious, cultural and economic influence, there is an upward trend in the consumption pattern of meat and meat-based products (Alahakoon et al., 2016). According to statistical data by DAPH (2014), the total meat production has increased from 119,620MT to $185,490 \mathrm{MT}$ in 2013. Sri Lanka exports poultry, beef, pork and mutton and poultry exports are recorded to be the highest (DAPH, 2013). Literature reveals that Sri Lanka imports meat from high meat producing countries over years. Poultry meat is the top imported type followed by mutton, beef, and pork (Alahakoon et al., 2016). Recent studies depict that still there is a shortage in the supply of poultry meat and other popular meat types in Sri Lanka (Alahakoon et al., 2016) and actions need to be taken to satisfy the consumer demand on meat.

With the increasing human population, demand for protein foods is also rising. Hence, it is a timely requirement to search for methods for protein production which are more sustainable, nutritious, with more emphasis on animal welfare (Stephans et al., 2018). Although the plant-based meat substitutes are favored by most meat consumers due to the health concerns, the usage is very low due to the less sensory acceptability (Ruzgys and Pickering 2020). The activists of cultured meat present this artificial meat as a potential substitute for consumers who want to be more responsible without changing their dietary pattern (Chriki and Hocquette, 2020; Ruzgys and Pickering, 2020). "In vitro meat production system is the production of meat outside the food animals by culturing the stem cells derived from farm animals inside the bioreactor by using advanced tissue engineering techniques" (Bhat et al., 2015). During culturing, extracted cells from farm animals are transferred to an appropriate medium containing nutrients, energy sources and other growth factors (Chriki and Hocquette, 2020) so that to promote the growth and differentiation of stem cells into mature muscle cells within a bioreactor (Welin, 2013). This involves the production of edible animal muscle by 
proliferating a small amount of muscle cells to a large portion of consumable flesh (Bhat et al., 2015). Even a living animal can be used to harvest muscle cells and cells from a single cow could produce 175 million quarter-pounders of meat whereas 440,000 cows are needed to produce the same amount of meat using conventional farming methods (Mancini and Antonioli, 2020).

Concerning the issues created by industrial animal farming the attention of scientists over the world has turned towards the research and development work on mass production of cultured meat (Rorheim et al., 2016). In-vitro meat production is anticipated to be resource efficient than conventional meat animal farming, especially concerning the demand for meat in future, more sustainable as the environmental impact will be low (Verbeke et al., 2015) with eliminating manure disposal problem and by reducing Green House Gas (GHG) emission (Rorheim et al., 2016; Ruzgys and Pickering 2020). It is estimated that cultured meat requires $7-45 \%$ less energy than conventional meat while $78-96 \%$ less emission of GHG, 99\% lesser land use and 82-96\% lower water usage (Welin, 2013). Cultured meat is safer than conventional meat considering human health aspects (Verbeke et al., 2015); as aseptic conditions are applied (Chriki and Hocquette, 2020) and antibiotics are not required during production process there is no risk of zoonotic infections (Rorheim et al., 2016). Furthermore, nutritional composition changes, for instance, the ratio of saturated fatty acids to polyunsaturated fatty acids (Chriki and Hocquette, 2020), can be applied on cultured meat during the production procedure which is non-applicable in conventional meat production. Regarding the animal welfare, unnecessary suffering imposed upon animals under human care and in factory farms and extremely brutal slaughtering of food animals are bypassed with this in-vitro meat production (Bhat et al. 2015; Verbeke et al., 2015; Rorheim et al., 2016). Nevertheless, even though large-scale production and market penetration results in a significant price reduction, extremely high unaffordable cost of cultured meat, increased requirement of technology and public acceptance of an unnatural meat are considered as the potential barriers for in-vitro meat production (Verbeke et al., 2015).

The current study aimed at unveiling the perception of Sri Lankans on cultured meat and their desire to try and buy if it becomes available in Sri Lankan context with the use of a questionnaire-based survey. The concept of lab-grown meat is totally a novel concept to Sri Lankans. Hence, a brief introduction including the pros and cons of production of in-vitro meat was delivered prior to presenting the pre-tested questionnaire. The major objective of this study was to analyze the perception of Sri Lankans about cultured meat, which is a very novel concept to Sri Lanka, their desire to accept in-vitro meat if it becomes available and the potential to introduce cultured meat to Sri Lanka. Accordingly, the survey focused on studying the meat consumption pattern among Sri Lankans, the extent of vegetarianism, whether there is a potential to introduce lab-grown meat to Sri Lanka, the important factors to be considered when introducing cultured meat to Sri Lankan society and the relationship of education level of people and the acceptance of this novel food technology.

\section{METHODOLOGY}

This survey was conducted using a sample of 14200 people covering 11 districts of Sri Lanka. A structural questionnaire was used for this survey.

\section{Sampling Procedure}

Probability sampling techniques were adopted to select sample for this survey. Eleven districts representing rural and urban communities in Sri Lanka were used for this survey and districts were used as clusters. Stratified random sampling and simple random sampling approaches were practiced to choose a sample of 14200 people for this survey. Based on the population 85 Divisional Secretariat divisions and 440 Gama Niladhari divisions were selected from these 11 districts using stratified random sampling technique and participants to the survey were selected by simple random sampling techniques from the selected Gama Niladhari divisions. The selected sample included individuals from various professional backgrounds such as medical, agricultural, engineering, banking, business, academic and administrative fields representing both government and private sector employees while $5 \%$ of the sample were nonemployed.

\section{Conducting the Questionnaire Based Survey}

The structured questionnaire was prepared. Questionnaire was designed with most closedended questions and few open-ended questions. Few open-ended questions were designed to obtain information about participant background information and to collect their opinion regarding the consumption of cultured-meat if it becomes available in Sri Lanka (i.e., the most considerable factor regarding the lab-grown meat). Close ended questions were in a double-choice or multiplechoice format and some questions demanded multiple answers while some questions allowed selecting more than one answer. Those questions mostly focused on quantitative data collection from the tested sample. Participants were not informed in advance to avoid biasness and modifications. The questionnaire was designed in the Sinhala, 
Tamil and English language. A brief outline, including the requirement of introducing in-vitro meat to modern societies and its pros and cons, was conducted prior to presenting the questionnaire. This survey was mainly conducted as an online survey mode using Google online survey platform Open-ended questions

The design of the questionnaire was composed of three sections: (1) socio-demographic information (gender, religion, residence; whether living in urban/ semi-urban/rural area, educational status, occupation and income level), (2) meat consumption pattern (i.e., whether participants are vegetarians or not, reasons for being vegetarians or meat consumers, preference on different meat types, the frequency of meat and meat-based product purchases and consumption at households) and (3) the perception of respondents on cultured meat (intention to reduce or avoid harvested meat, intention to purchase or consume lab-grown meat if it becomes available, factors consider when purchasing cultured meat, whether vegetarians are prone to consume cultured meat, whether meat consumers are prone to substitute harvested meat with cultured meat and whether the participants agree with six important facts; the first three statements were regarding the positive impacts of cultured meat production process (i.e., the involvement in environmental sustainability, food security and animal welfare), the remaining three statements were about beliefs regarding the intrinsic characteristics (i.e., cultured meat would be similar or better in safety aspects, sensory attributes, and nutritional aspects). To evaluate the level of agreement on the above facts, the participants were provided with a five-point Likert scale, ranging from "strongly disagree" (1) to "strongly agree" (5). The relationship between the educational levels of people with the possibility for acceptance of lab-grown meat concept by consumers was tested using regression analysis. The education level of participants was taken as 1.0: Primary education; 2.0: Secondary Education and 3.0: University Education. Their desire to purchase lab-grown meat was ranked using Likert scale ranging from 1-3; 1: Never, 2: May be and 3: Yes, will try. Minitab14 software program was employed for the statistical analysis of collected data (non-parametric) while some data was descriptively analyzed using Microsoft Excel 2010.

\section{RESULTS AND DISCUSSION}

\section{Socio-demographic information of the tested sample}

The questioned sample was comprised of $67.5 \%$ men and $32.5 \%$ women. Other socio-demographic information of the survey is shown in Table 1.
Table 1: Socio-demographic information

\begin{tabular}{lc}
\hline \multicolumn{2}{c}{ Age (years) } \\
\hline$<25$ & $8.75 \%$ \\
$25-45$ & $62.5 \%$ \\
$46-65$ & $15.00 \%$ \\
$>65$ & $13.75 \%$ \\
\hline \multicolumn{2}{c}{} \\
\hline Buddhist & $85.00 \%$ \\
Catholic & $3.75 \%$ \\
Muslim & $6.25 \%$ \\
Hindu & $3.75 \%$ \\
Other & $1.25 \%$ \\
\hline \multicolumn{2}{c}{} \\
\hline Urban & $30.00 \%$ \\
Semi urban & $46.25 \%$ \\
Rural & Place of residence \\
\hline
\end{tabular}

Generally, education level and income of the community are very important parameters when studying a perception (Wilks and Phillips, 2017) and following Figures (Figure 1 and 2) depict the education level and income level of the survey group.

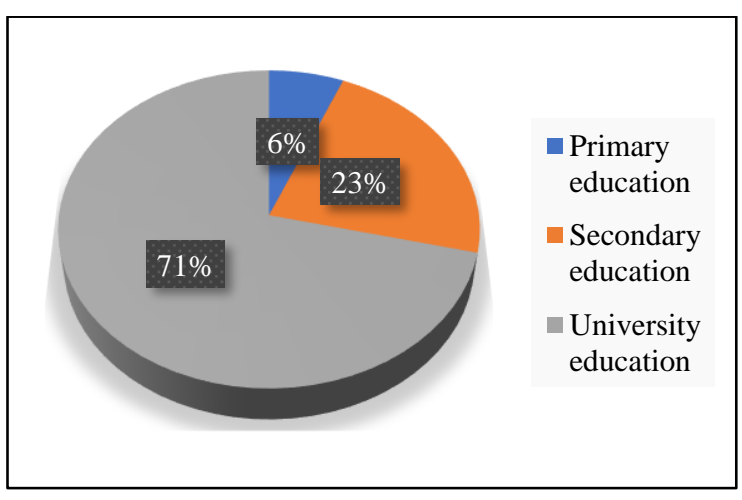

Figure 1: Education levels of the survey group

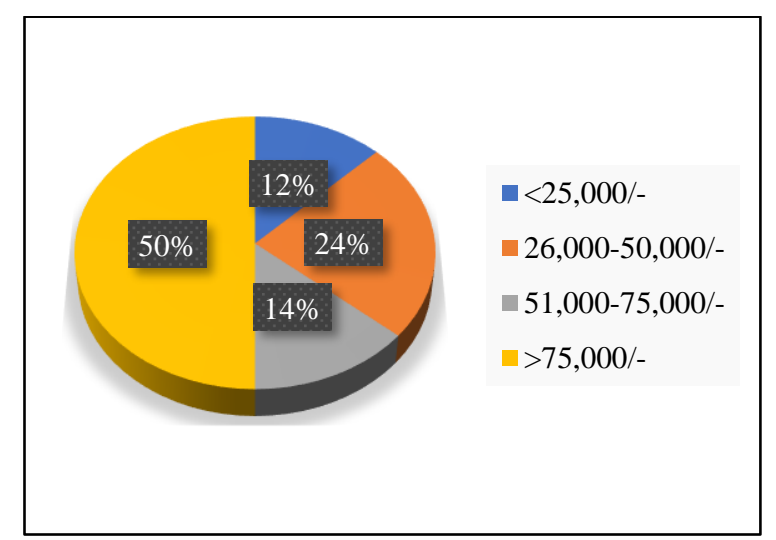

Figure 2: Income levels (LKR) of the survey group 


\section{Consumption pattern}

Only $2 \%$ of the sample was recorded to be vegetarians who avoid meat products. The reasons for being vegetarians, was the unwillingness towards animal slaughtering, health concerns and religious concerns and $71 \%$ out of them expressed willingness to consume cultured meat. Literature revealed that the production of in-vitro meat is acceptable and worth promoting concerning the vegetarian concepts (Wilks and Phillips, 2017; Stephans et al., 2018). Those who consume meat, $38.4 \%$ stated their intention to reduce conventional meat consumption and all those were females. As a whole, this fact proves that consumers are hesitant to cut their meat consumption in a substantial way although some evidence declared ongoing interest among Sri Lankans in reducing meat consumption by considering the negative health impacts related with conventional meat consumption.

When considering the meat consumption pattern of the tested group of people, chicken meat was noted as the most preferred meat item which was followed by the pork, mutton, and beef respectively. Desire on different meat types were recorded as; chicken $86.25 \%$, pork $50 \%$, mutton $45 \%$ and beef $30 \%$. Only $10.61 \%$ of the interviewees consume meat products in all main meals and $69.7 \%$ of the group consume meat frequently but not in all main meals. Among the tested participants, $13.64 \%$ consume meat only in special meals while $6.06 \%$ consume meat rarely. Further, $24.25 \%$ of the sample purchases raw meat twice or more per week, $48.5 \%$ purchase around once a week and the rest purchase once a month. And, $56.06 \%$ mentioned that they purchase $1-5 \mathrm{~kg}$ in each time whereas $43.94 \%$ said that it is less than $1 \mathrm{~kg}$. Regarding the processed meat products consumption, $38.46 \%$ of the tested sample commented that they purchase processed meat products such as sausages, meat balls once a week whereas $23.08 \%$ stated that they purchase once a month.

\section{Perception on cultured meat}

In current study, the statistical evaluation of factors considered by the respondents when they are purchasing cultured meat (when it is available) revealed that the impact on health is the highest considered factor followed by nutritional properties, sensory properties, and price (KruskalWallis test, average rank 160.5). The $75 \%$ of the sample had displayed an interest towards the novel product. Thus, it is possible to predict that there will be a substantial market in Sri Lanka for cultured meat especially after making consumers more educated regarding this concept. But it will remain indefinite how the consumers would react to this novel food produced utilizing a different technology compared to prevailing livestock methods as well as under which circumstances, they would accept this innovative food (Verbeke $e t$ al., 2015; Stephans et al., 2018). Hence, it can be recommended that there is a requirement to conduct some more research surveys in Sri Lankan context regarding this novel concept.

The statistical analysis (Wilcoxon Signed Rank Test) confirmed that the tested sample agrees with the first three statements which state cultured meat supports to preserve natural resources $(p<0.05)$, supports better animal welfare $(p<0.05)$ and contributes to alleviate starvation $(\mathrm{p}<0.05)$. The results revealed that the consumers understand the negative impacts of intensive animal farming (for meat) on sustainable environmental aspects and animal welfare. In addition, production of cultured meat can contribute to ensure food security of the country.

Results suggested that there was no evidence to accept that the tested sample agrees on statements which state cultured meat is similar or better in sensory $(p>0.05)$ and nutritional properties $(p>0.05)$ to that of harvested meat and cultured meat is safe $(p>0.05)$ to consume. To test the consumer acceptability of cultured meat, sensory attributes of meat, for instance, flavor, tenderness and juiciness, must be evaluated with a sound analytical procedure. Especially when considering meat substitutes, consumers are not ready to accept a new substitute rapidly as they search for the meaty flavor and other specific sensory attributes of natural meat to reduce the food neophobia (Mancini and Antonioli, 2020). People with food neophobia are prone to reject consumption of cultured meat (Ruzgys and Pickering, 2020). Concerning the nutritional aspects, it is of crucial importance to provide detailed nutritional facts on the developed novel product to consumers as it is the second most considered intrinsic attribute. Regarding the safety aspect of cultured meat, people need to know in which way the product is not making any harm to human health majorly due to the fact that consumers view cultured meat as an unnatural product which is one of the strongest obstacles for the public acceptance of this product (Welin, 2013).

Previous studies disclosed that lack of trust, uncertainty, and concerns over potential adverse long-term consequences are resulted by the unfamiliarity with novel technologies (Frewer et al., 2013; Marcu et al., 2015; Siegrist and Sütterlin, 2017). Even though, there is great uncertainty regarding the acceptance of cultured meat by consumers, provision of information and educating consumers is an effective way to inspire the public to express their opinions and be more accepting (Palmieri et al., 2021). 
A regression analysis was conducted to test the relationship between education level and the desire on purchasing laboratory grown meat confirmed that the acceptance level of the lab-grown meat increases with the education level of consumers (Figure 3). Accordingly, it was revealed that people with better education would be the potential group of consumers of cultured meat when it will be available in Sri Lankan context. However, the acceptance of cultured meat can be further raised by awareness and education programs among the consumers in an organized manner, (Palmieri et al., 2021) even among the consumers with lower educational levels.

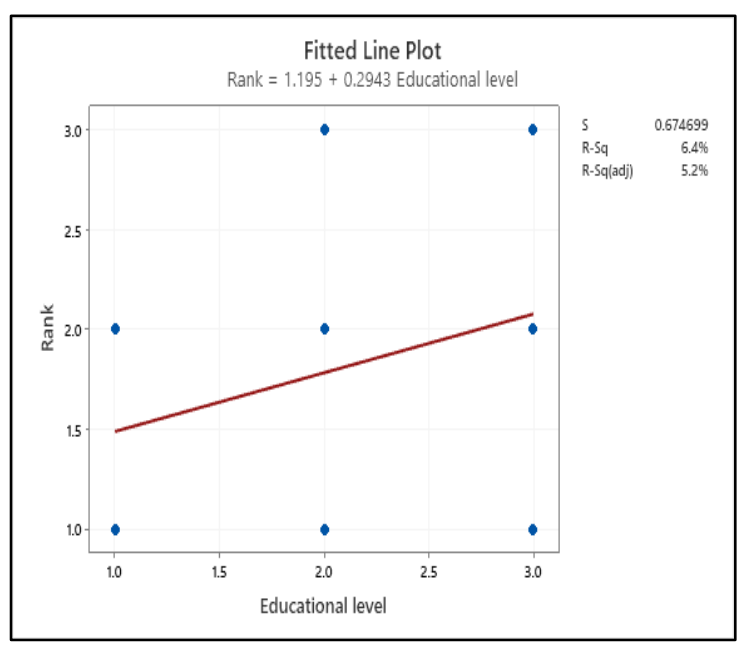

Figure 3: Relationship between education level of consumers and acceptance of cultured meat. Education level; 1.0: Primary education; 2.0: Secondary Education; 3.0: University Education, Rank; Likert scale to depict the desire to purchase cultured meat; 1: Never, 2: May be and 3: Yes, will try.

Answer of most respondents for the question "most considered factor when purchasing labgrown meat" showed, that genetically modified food is the most considered factor. Hence, cultured meat with genetic modifications is probable to face more resistance from the public when compared with non-genetically modified cultured meat.

\section{CONCLUSIONS}

This study basically assessed the meat consumption pattern of Sri Lankans in addition to the evaluation of their perception on cultured meat. It was revealed that Sri Lankan meat consumers are reluctant to cut their meat consumption in a substantial amount even though people are more informed about the negative health impacts of continuous consumption of conventional meat. Further the study recognized that a satisfactory count of consumers including both vegetarians and non-vegetarians were interested in experiencing cultured meat product. There was a good acceptance level for the lab-grown meat in Sri Lanka if meat is not genetically modified. In addition to that, the level of acceptance of this novel product has linear correlation with the education level of the consumers. These results confirmed that Sri Lankans have a good understanding about the positive impacts of cultured meat on animal welfare, environmental sustainability and food security. The survey recommended the requirement of further investigations on this concept which is a novel concept to Sri Lanka..

\section{REFERENCES}

Alahakoon, A.U., Jo, C. and Jayasena, D.D. 2016. An Overview of Meat Industry in Sri Lanka: A Comprehensive Review. Korean Journal for Food Science of Animal Resources, 36(2): 137-144. DOI: 10.5851/kosfa.2016.36.2.137

Bhat, Z.F., Kumar, S. and Fayaz, H. 2015. In vitro meat production: Challenges and benefits over conventional meat production. Journal of Integrative Agriculture, 14(2): 241-248. DOI: 10.1016/S2095-3119(14)60887-X

Chriki, S. and Hocquette, J.F. (2020 The Myth of Cultured Meat: A Review. Front. Nutr, 7:7. DOI: $10.3389 /$ fnut.2020.00007

Department of Census and Statistics. 2012. Census of Population and Housing -2012 Sri Lanka. Available at: http://www.statistics.gov.lk/pophousat/cph201 1/pages/activities/reports/finalreport/populatio n/finalpopulation.pdf (Accessed: 08 September 2021).

Frewer, L.J., Van der Lans, I.A., Fischer, A.R.H., Reinders, M.J., Menozzi, D., Zhang, X., Vanden-Berg, I. and Zimmermann, K.L. 2013. Public perceptions of agri-food applications of genetic modification-A systematic review and meta-analysis. Trends Food Sci. Technol, 30:142-152. DOI: 10.1016/j.tifs.2013.01.003

Holmand, L. and MÖhl, M. 2000. The role of meat in everyday food culture: an analysis of an interview study in Copenhagen. Appetite, 34:277-283. DOI:10.1006/appe.2000.0314

Mancini, M.C. and Antonioli, F. 2020. To what extent are consumers' perception and acceptance of alternative meat production systems affected by information? The case of cultured meat. Animals, 10(656). DOI: 10.3390/ani10040656

Marcu, A., Gaspar, R., Rutsaert, P., Seibt, B., Fletcher, D., Verbeke, W. and Barnett, J. 2015. Analogies, metaphors, and wondering about the future: Lay sense-making around synthetic meat. Public Understing of Science, 24: 547562. DOI: $10.1177 / 0963662514521106$ 
Palmieri, N., Perito, M.A. and Lupi, L. 2021. Consumer acceptance of cultured meat: some hints from Italy, British Food Journal, 123(1): 109-123. DOI: 10.1108/BFJ-02-2020-0092

Rorheim, A., Mannino, A., Baumann, T. and Caviola, L. 2016. Cultured Meat: An Ethical Alternative to Industrial Animal Farming. Policy paper by Sentience Politics, 1: 1-14.

Ruzgys, S. and Pickering, G.J. (2020. Perceptions of cultured meat among youth and messaging strategies. Frontiers in sustainable food systems, $4(122)$ : $\quad$ DOI: $10.3389 /$ fsufs. 2020.00122

Siegrist, M. and Sütterlin, B. 2017. Importance of perceived naturalness for acceptance of food additives and cultured meat. Appetite, 113:320-326.

DOI: 10.1016/j.appet.2017.03.019

Stephens, N., Silvio, L.D., Dunsford, I., Ellis, M., Glencross, A. and Sexton, A. 2018. Bringing cultured meat to market: Technical, sociopolitical, and regulatory challenges in cellular agriculture. Trends in Food Science and Technology, 78: 155-166. DOI: 10.1016/j.tifs.2018.04.010

Verbeke, W., Pierre, S. and Loo, E.J.V. 2015. Challenges and prospects for consumer acceptance of cultured meat. Journal of Integrative Agriculture, 14(2): 285-294. DOI:10.1016/S2095-3119(14)60884-4

Welin, S. 2013. Introducing the new meat. Problems and prospects. Etikk I PraksisNordic Journal of Applied Ethics, 7(1): 2437. DOI: $10.5324 /$ eip.v7i1.1788

Wilks, M. and Phillips, C.J.C. 2017. Attitudes to in vitro meat: A survey of potential consumers in the United States. PLoS ONE, 12(2) DOI: $\underline{10.1371 / \text { journal.pone.0171904 }}$ 